\title{
Correction: GNE myopathy in Chinese population: hotspot and novel mutations
}

\author{
Yang Chen ${ }^{1}$ Jianying $\mathrm{Xi}^{1} \cdot$ Wenhua $\mathrm{Zhu}^{2} \cdot$ Jie Lin $^{1} \cdot$ Sushan Luo ${ }^{1} \cdot$ Dongyue Yue $^{3}$. Shuang Cai ${ }^{1}$. Chong Sun ${ }^{1}$. \\ Chongbo Zhao ${ }^{1,3} \cdot$ Satomi Mitsuhashi ${ }^{4,5} \cdot$ Ichizo Nishino $^{4,5} \cdot$ Minjie Xu $^{6} \cdot$ Jiahong $\mathrm{Lu}^{7,8}$
}

Published online: 26 December 2018

(c) The Author(s) under exclusive licence to The Japan Society of Human Genetics 2018

Correction to: Journal of Human Genetics https://doi.org/ 10.1038/s10038-018-0525-9; published online: 2 November 2018;

Since the publication of the above article, the authors of the above paper have noticed errors in the affiliation information. The affiliation information is provided correctly, below. The authors would like to apologize for this mistake.

Wenhua Zhu

whzhu@fudan.edu.cn

$\triangle$ Jiahong Lu

lujiahong@fudan.edu.cn

1 Department of Neurology, Huashan Hospital, Fudan University, Shanghai, China

2 Department of Neurology, Huashan Hospital, Fudan University, Shanghai, China

3 Department of Neurology, Jing'an District Center Hospital of Shanghai, Fudan University, Shanghai, China

4 Department of Neuromuscular Research, National Institute of Neuroscience, National Center of Neurology and Psychiatry (NCNP), Tokyo, Japan

5 Department of Genome Medicine Development, Medical Genome Center, National Center of Neurology and Psychiatry (NCNP), Tokyo, Japan

6 Key Laboratory of Contraceptives and Devices, Shanghai Institute of Planned Parenthood Research, Institute of Reproduction and development, Fudan University, Shanghai, China

7 Department of Neurology, Huashan Hospital, Fudan University, Shanghai, China

8 Institute of Neurology, Huashan Hospital, Fudan University, Shanghai, China 